\title{
Criminologie
}

\section{Le climat social dans les institutions pour jeunes délinquants}

\section{Robert Ménard et Marc LeBlanc}

Volume 11, numéro 1, 1978

Centres d’accueil du Québec : accréditation et évaluation

URI : https://id.erudit.org/iderudit/017080ar

DOI : https://doi.org/10.7202/017080ar

Aller au sommaire du numéro

Éditeur(s)

Les Presses de l'Université de Montréal

ISSN

0316-0041 (imprimé)

1492-1367 (numérique)

Découvrir la revue

Citer cet article

Ménard, R. \& LeBlanc, M. (1978). Le climat social dans les institutions pour jeunes délinquants. Criminologie, 11(1), 7-23. https://doi.org/10.7202/017080ar d'utilisation que vous pouvez consulter en ligne.

https://apropos.erudit.org/fr/usagers/politique-dutilisation/ 
LE CLIMAT SOCIAL DANS LES INSTITUTIONS POUR JEUNES DÉLINQUANTS

Robert Ménard

Marc LeBlanc

\section{LE CLIMAT SOCIAL EN INSTITUTION}

Le Groupe de recherche sur l'inadaptation juvénile de l'Université de Montréal a entrepris depuis 1973 des recherches évaluatives en milieu institutionnel pour juvéniles. Dans le cadre de ces recherches, la mesure du climat social de trois institutions québécoises a été évaluée à l'aide de l'instrument "Correctional Institutions Environment Scale " développé par Rudolf H. Moss (1973).

S'il est vrai que l'environnement d'un milieu donné influence l'attitude et le comportement des membres de ce milieu; connaître le climat social régnant dans une institution pour jeunes délinquants semble un élément important et utile au travail rééducatif effectũé auprès de ceux-ci. Dans le présent article, le climat social existant à l'intérieur de trois institutions québécoises est décrit et comparé à celui de certaines institutions américaines et yougoslaves; ceci afin de dégager certaines caractéristiques d'un climat social propice à la rééducation et afin, dans un deuxième temps, de voir de quelles utilités cliniques et administratives peut être la connaissance du climat social dans un milieu institutionnel.

\section{Problématique}

L'environnement influence les individus et leur comportement. Une telle assertion concernant le comportement des individus mérite certes l'attention. L'influence d'un logis surpeuplé, la contamination criminelle dans les prisons sont autant de points sur lesquels se sont penchés les chercheurs afin de comprendre et d'expliquer le comportement des individus.

En fait, on attache de plus en plus d'importance à l'étude des influences situationnelles et environnementales agissant sur le comportement humain. Plusieurs travaux dont ceux de Mischel (1963), Magnusson, Gerzen et Nyman (1968) et Moos (1969) démontrent que les influences environnementales peuvent jouer 
une part plus importante, dans la détermination du comportement individuel, qu'on aurait pu le croire dans le passé.

L'analyse systématique et la description des caractéristiques psycho-sociales de différents types d'environnement furent, entre autres, l'objet de nombreux travaux entrepris par Moos et ses associés (Gerst et Moos, 1972, Wenk et Moos 1972, Moos 1973, Moos et Humphrey, 1973). Ces différentes études visent à évaluer le climat social existant dans un environnement donné, c'est-à-dire dans un groupe donné de gens. Des dimensions telles que la cohésion des pairs, l'expression, l'autonomie des membres du groupe sont investiguées dans ces études des caractéristiques psycho-sociales et du climat organisationnel.

De nombreuses études criminologiques se sont intéressées à définir le climat social le plus propice à la rééducation. L'ensemble des auteurs, dont C.F. Jesness (1962) et M. Capul (1969), conviennent qu'un contrôle rigide crée un climat qui est à toute fin pratique l'antithèse d'un milieu de vie thérapeutique.

Un régime de vie reposant sur l'autoritarisme et le contrôle, c'est-à-dire dominé par la surveillance, la discipline et l'intolérance des éducateurs, est donc un milieu où l'on s'attarde plus à faire respecter l'ordre qu'à comprendre et à amener les jeunes à modifier leur comportement. Un tel milieu voit son atmosphère s'imprégner d'aggressivité et d'hostilité. Rapidement, le milieu est emprisonné dans un cercle vicieux; face à l'autoritarisme les jeunes se sentent menacés et développent un système de défense, et plus le système de défense s'accentue et menace l'ordre, plus le contrôle est renforcé.

A l'opposé, un régime trop permissif et un libéralisme démesuré conduisent à un "laissez-faire » de la part des éducateurs, à un abandon de leur rôle de contrôle qui entraîne l'instabilité et favorise la domination des éléments les plus agressifs et antisociaux (Selosse et al., 1972).

Le système à instaurer est un système démocratique caractérisé par la mise en commun consciente des ressources des éducateurs et des jeunes et promouvant la participation active de ceux-ci ; le tout peut se traduire par le partage des responsabilités et des prises de décisions, l'existence d'une ambiance tolérante, d'une information diffusée et l'expression libre des sentiments. Empey et Lubeck (1971) soutiennent que la motiva- 
tion du jeune à changer, intérieurement et extérieurement, sera accrue s'il peut devenir un participant actif dans la solution de ses propres problèmes et de ceux de ses pairs; s'il baigne dans une ambiance de confiance et de solidarité.

\section{Instrument}

Partant de ces attentes des criminologues en regard de la nature du climat propice à la rééducation, nous nous sommes demandé si celui-ci caractérisait certaines institutions québécoises. Pour ce faire nous avons sélectionné l'instrument de Moos (1973) : le "Correctionel Institution Environment Scale 》. Il s'agit d'un questionnaire de 86 item auxquels on répond par vrai ou faux.

Ce questionnaire a été validé par des travaux en Yougoslavie (Vodopivec, 1974), aux États-Unis (Moos, 1973), au Québec (Ménard, 1976). Il ressort qu'il s'agit d'un instrument stable dans le temps, fidèle et valide selon les données intensives rapportées par Moos (1973). Il a de plus l'avantage de pouvoir permettre des comparaisons transculturelles.

Les 86 item du questionnaire se distribuent sur neuf échelles et trois grandes dimensions et les scores varient de 1 à 10 sur chacune des échelles. Le tableau I présente la définition des échelles.

\section{TABLEAU I}

Correctional Institutions Environment Scale (R.H. Moos, 1973)

Instrument traduit et adapté par la recherche Evaluation de Boscoville

1. Engagement : Mesure jusqu'à quel point les jeunes sont actifs et énergiques dans leur conduite quotidienne à travers le programme; c'est-àdire la façon dont ils agissent socialement avec les autres jeunes, l'initiative dont ils font preuve et le développement d'une fierté et d'un esprit de groupe dans le programme.

2. Support: Mesure principalement le niveau de support des jeunes par les éducateurs et, à un degré moindre, le support mutuel des jeunes.

3. Expression: Mesure le degré d'incitation du programme à l'expression ouverte des sentiments (incluant les sentiments d'agressivité) par les jeunes.

4. Autonomie: Mesure le degré d'encouragement des jeunes à prendre des initiatives dans la planification des activités.

5. Orientation pratique: Mesure jusqu'à quel point l'environnement du jeune l'oriente à se préparer lui-même à sa libération de l'institution.

6. Orientation des problèmes personnels: Mesure le degré d'encouragement des jeunes à s'occuper de leurs problèmes personnels et de leurs sentiments et à chercher à se comprendre entre eux. 
7. Ordre et organisation: Mesure l'importance de l'ordre et de l'organisation dans le programme, au niveau de la façon dont c'est perçu par les jeunes, de ce que font les éducateurs pour encourager l'ordre et la facilité avec laquelle c'est maintenu.

8. Clarté: Mesure jusqu'à quel point les jeunes savent à quoi s’attendre dans la routine quotidienne de leur programme, et le degré d'explication des règles et procédures du programme.

9. Contrôle des éducateurs: Mesure jusqu'à quel point les éducateurs utilisent des mesures pour maintenir les jeunes sous contrôle, c'est-àdire dans la formulation des règles, dans la cédulation des activités et dans les relations entre les jeunes et les éducateurs.

\section{Le climat social dans les institutions pour juvéniles délinquants}

\subsection{Institutions américaines et québécoises}

Le tableau II représente les moyennes ' enregistrées aux neuf échelles dans 28 institutions américaines et dans les trois institutions québécoises; ces moyennes traduisent la perception des jeunes et des éducateurs du climat social dans le milieu de

\section{TABLEAU II}

Le climat social dans les institutions québécoises et américaines (Moyennes et rangs)

\begin{tabular}{|c|c|c|c|c|c|c|c|c|}
\hline & \multicolumn{4}{|c|}{ Institutions américaines } & \multicolumn{4}{|c|}{ Institutions québécoises } \\
\hline & \multicolumn{2}{|c|}{$\begin{array}{l}\text { Jeunes } \\
\text { (3651) }\end{array}$} & \multicolumn{2}{|c|}{$\begin{array}{l}\text { Educateurs } \\
\text { (858) }\end{array}$} & \multicolumn{2}{|c|}{$\begin{array}{l}\text { Jeunes } \\
(252)\end{array}$} & \multicolumn{2}{|c|}{$\begin{array}{l}\text { Educateurs } \\
\text { (128) }\end{array}$} \\
\hline & Moy & tang & Moy. & lang & Moy. & Rang & Moy. & lang \\
\hline Engagement & 4,74 & 5 & 6,95 & 4 & 6,56 & 4 & 7,45 & 5 \\
\hline Support des éducateurs & 5,13 & 3 & 7,32 & 2 & 7,43 & 1 & 8,32 & 2 \\
\hline Expression & 4,38 & 7 & 5,82 & 8 & 5,45 & 7 & 7,44 & 6 \\
\hline Autonomie & 4,12 & 9 & 6,79 & 6 & 6,22 & 6 & 7,32 & 7 \\
\hline Orientation pratique & 6,08 & 2 & 7,45 & 1 & 7,09 & 2 & 7,99 & 3 \\
\hline $\begin{array}{l}\text { Orientation des } \\
\text { problèmes personnels }\end{array}$ & 4,44 & 6 & 7,03 & 3 & 5,41 & 8 & 6,15 & 8 \\
\hline Ordre et organisation & 4,32 & 8 & 6,09 & 7 & 6,39 & 5 & 7,47 & 4 \\
\hline Clarté & 5,12 & 4 & 6,89 & 5 & 6,97 & 3 & 8,43 & 1 \\
\hline Contrôle des éducateurs & 6,91 & 1 & 5,55 & 9 & 5,18 & 9 & 4,15 & 9 \\
\hline
\end{tabular}

1. Le minimum possible est 0 et le maximum 10 . 
vie institutionnelle. La population étudiée par Moos se compose de 3651 jeunes et 858 éducateurs ou membres du personnel alors que 252 jeunes et 128 éducateurs constituent les groupes québécois. Une remarque s'impose : la population américaine est plus hétérogène que la population québécoise. En effet, la population américaine comporte un nombre plus élevé de sujets, une provenance plus diversifiée et un certain nombre de filles, ce qui n'est pas le cas dans l'échantillon québécois.

Il ressort de cette comparaison qu'un fort écart de perception du climat social existe entre les jeunes québécois et les jeunes américains. Sauf à l'échelle * contrôle des éducateurs », les jeunes des institutions américaines ont des moyennes inférieures aux jeunes des institutions québécoises et ceci généralement de l'ordre de deux points.

$\mathrm{Si}$ on fait l'hypothèse que le climat perçu par les jeunes dépeint dans une certaine mesure la philosophie ou la politique rééducative suivie par les institutions qui les accueillent, on peut avancer que les institutions américaines mettent principalement l'accent sur le contrôle des jeunes par les éducateurs et incitent les jeunes à préparer concrètement leur départ de l'institution : ce sont les échelles dont les moyennes sont les plus élevées. Tout l'aspect traitement ou ambiance thérapeutique ne semble pas être perçu par les jeunes. En effet, on ne semble pas les encourager à s'engager dans un progranme rééducatif, à les amener à se comprendre et à changer intérieurement, à être autonome. Ce sont les échelles dont les moyennes sont les plus faibles.

La perception des jeunes des institutions québécoises indique que non seulement l'accent n'est pas placé sur le contrôle des éducateurs, mais ce contrôle est assez faible comparé aux autres orientations thérapeutiques : les moyennes sont faibles sur le contrôle et fortes sur le changement. Les jeunes perçoivent leur milieu comme un endroit où on les oriente vers l'avenir comme dans les institutions américaines, mais ça ne se résume pas uniquement à ce point. Ils ont également l'impression d'être fortement supportés par les éducateurs et d'être encouragés à s'engager dans leur programme rééducatif, qu'ils perçoivent comme étant bien maîtrisé par les éducateurs et clair dans ses règles et procédures.

Précédemment, nous avons cité certains auteurs prônant un contrôle souple face aux jeunes en institution, un régime trop 
libéraliste ou trop autoritaire pouvant avoir des effets néfastes. Sans être des extrêmes, les résultats différents enregistrés dans les institutions américaines et québécoises tendent à appuyer la nécessité d'un contrôle souple. Par ailleurs, il ressort du tableau II que le contrôle des éducateurs est fonction d'autres facteurs insérés dans un programme de rééducation; suivant que l'on mettra l'accent ou non sur ces facteurs, l'aspect « contrôle » pourra varier en intensité ; Ménard (1976) rapporte à cet égard des corrélations négatives entre le contrôle et l'ambiance thérapeutique. Le fait que les éducateurs réussissent à engager les jeunes dans leur programme, que l'ordre et l'organisation à l'intérieur du programme soient bien implantés, que les règles et procédures soient bien comprises et que le personnel accorde un bon support aux jeunes sont possiblement des facteurs qui compensent avantageusement la nécessité d'un contrôle strict de la part du personnel éducatif. En somme, nous pourrions résumer en parlant de la capacité d'intégration de l'institution, que plus une institution réussit à impliquer le jeune et à l'engager face à son milieu et dans son programme rééducatif, moins il est nécessaire d'appliquer un contrôle rigide.

Les éducateurs ont également répondu au questionnaire afin de voir la concordance de perception des éducateurs et des jeunes relativement au milieu de vie de ces derniers. Le tableau II fait ressortir une différence de perception au niveau de l'intensité. En effet, autant les éducateurs des institutions américaines que ceux des institutions québécoises affichent des moyennes plus élevées que celles des jeunes aux diverses échelles; les écarts sont d'un à deux points entre ces groupes. La seule exception se situe à l'échelle «contrôle des éducateurs », les éducateurs percevant moins de contrôle. Cependant, si les deux groupes d'institutions sont comparés, on remarque que l'écart de perception est dans l'ensemble plus grand entre les jeunes et les éducateurs des institutions américaines. Dans ces dernières, non seulement l'écart est plus grand, mais si on ordonne les échelles par ordre de grandeur, à partir des moyennes, d'autres différences ressortent. Ainsi, les jeunes avec une moyenne de 4,12 à l'échelle autonomie relèguent cette échelle en dernière position, soit au neuvième rang. Par contre, la moyenne des éducateurs $(6,79)$ classe cette échelle au sixième rang. Un même écart de rang se retrouve à l'échelle orientation des problèmes personnels, soit le rang 6 chez les 
jeunes et le rang 3 chez les éducateurs. Toutefois, l'échelle où s'opposent le plus catégoriquement les jeunes et les éducateurs est l'échelle « contrôle des éducateurs ». En effet, les jeunes avec une moyenne de 6,91, classent cette échelle au premier rang, alors que les éducateurs avec une moyenne de 5,55 la relèguent au dernier rang. Donc, une nette différence de perception existe entre les deux groupes. Des différences aussi marquées ne se retrouvent pas dans les institutions québécoises. Par exemple, l'échelle contrôle des éducateurs se situe au dernier rang autant chez les jeunes que chez les éducateurs, avec respectivement des moyennes de 5,18 et 4,15 .

En somme, outre une dissimilitude de perception assez marquée dans les institutions américaines, on note aussi que l'échelle contrôle s'avère ici également un facteur d'intérêt lorsqu'il y a comparaison entre jeunes et éducateurs, ce fait tend à confirmer l'attention qui doit être accordée à ce facteur.

\subsection{Institutions yougoslaves et québécoises}

Le tableau III présente la perception des jeunes et des éducateurs de trois institutions yougoslaves et des trois institutions québécoises. Les trois institutions yougoslaves sont celles étudiées par Katja Vodopivec (1974), et les résultats sur le climat social reproduits au tableau III sont ceux recueillis au Québec ${ }^{2}$ à l'aide de l'instrument développé par Moos.

L'institution Radece présente dans l'ensemble les plus faibles moyennes. Suivent les institutions Slivnica, Boys' Farm et Mont Saint-Antoine, lesquelles ont des moyennes similaires, et finalement les institutions Logatec et Boscoville, cette dernière affichant les plus fortes moyennes. L'échelle contrôle des éducateurs permet d'entrevoir la nature du régime de vie perçu dans chaque institution. A l'institution Radece, les jeunes perçoivent un contrôle assez élevé en comparaison avec les autres institutions : la moyenne est de 6,22 contre 4 et 5 pour les autres institutions. De fait, la deuxième plus forte moyenne est enregistrée à l'échelle contrôle des éducateurs à Radece alors que dans les autres institutions cette échelle se situe au dernier ou dans les derniers rangs.

2. Les données québécoises proviennent de trois recherches différentes dirigées, par Marc LeBlanc, à Boscoville, Boys' Farm et au Mont SaintAntoine. 
TABLEAU III

Le climat social dans les institutions québécoises et yougoslaves (Moyennes)

\begin{tabular}{|c|c|c|c|c|c|c|c|c|c|c|c|c|}
\hline \multirow[b]{3}{*}{ Nombre de sujets } & \multicolumn{2}{|c|}{ Radece } & \multicolumn{2}{|c|}{ Slivnica } & \multicolumn{2}{|c|}{ Logatec } & \multicolumn{2}{|c|}{ Boys' Farm } & \multicolumn{2}{|c|}{ Mont St-Antoine } & \multicolumn{2}{|c|}{ Boscoville } \\
\hline & Jeunes & $\begin{array}{l}\text { Educa- } \\
\text { teurs }\end{array}$ & Jeunes & $\begin{array}{l}\text { Educa- } \\
\text { teurs }\end{array}$ & Jeunes & $\begin{array}{l}\text { Educa- } \\
\text { teurs }\end{array}$ & Jeunes & $\begin{array}{c}\text { Educa- } \\
\text { teurs }\end{array}$ & Jeunes & $\begin{array}{c}\text { Educa- } \\
\text { teurs }\end{array}$ & Jeunes & $\begin{array}{l}\text { Educa- } \\
\text { teurs }\end{array}$ \\
\hline & 94 & 28 & 54 & - & 38 & 18 & 53 & 36 & 160 & 62 & 42 & 31 \\
\hline Engagement & 4,64 & 4,29 & 6,10 & - & 6,19 & 6,06 & 6,22 & 8,25 & 6,34 & 6,44 & 7,81 & 8,55 \\
\hline Support des éducateurs & 5,68 & 6,31 & 7,02 & - & 7,81 & 8,56 & 7,02 & 8,86 & 7,17 & 7,52 & 8,93 & 9,29 \\
\hline Expression & 4,22 & 5,16 & 4,36 & - & 6,33 & 7,78 & 5,51 & 7,87 & 5,05 & 6,81 & 6,98 & 8,21 \\
\hline Autonomie & 5,86 & 6,03 & 6,60 & - & 7,53 & 8,89 & 6,40 & 8,12 & 5,89 & 6,76 & 7,28 & 7,46 \\
\hline Orientation pratique & 6,51 & 7,32 & 6,74 & 一 & 7,39 & 8,19 & 6,98 & 8,75 & 6,80 & 7,34 & 8,33 & 8,36 \\
\hline $\begin{array}{l}\text { Orientation des } \\
\text { problèmes personnels }\end{array}$ & 5,86 & 7,00 & 6,50 & - & 7,62 & 7,99 & 5,33 & 7,01 & 5,24 & 5,56 & 6,11 & 6,34 \\
\hline Ordre et organisation & 5,25 & 3,70 & 5,72 & 一 & 5,37 & 2,53 & 5,72 & 6,50 & 6,22 & 7,26 & 7,86 & 9,07 \\
\hline Clarté & 5,86 & 5,93 & 6,30 & 一 & 7,00 & 7,17 & 6,52 & 7,78 & 6,64 & 8,19 & 8,76 & 9,61 \\
\hline Contrôle des éducateurs & 6,22 & 5,43 & 5,30 & - & 4,17 & 2,10 & 5,20 & 4,23 & 5,40 & 3.83 & 4,29 & 4,70 \\
\hline
\end{tabular}


Un certain rapport semble exister entre l'échelle contrôle et l'échelle support. Disposées en un continuum, les moyennes enregistrées à ces deux échelles, dans les six institutions, présentent une relation inverse. Ainsi, Radece renferme le plus fort contrôle mais le plus faible support. Inversement, à Logatec et Boscoville, où le contrôle est plus faible que dans les quatre autres institutions, le support est le plus élevé. À un fort contrôle correspond donc un faible support.

Dans toutes les institutions, sauf à l'institution Slivnica où nous ne disposons pas de l'évaluation des éducateurs, ceux-ci affichent sur la majorité des échelles des moyennes supérieures à celles des jeunes. Cette seule observation n'est pas en soi alarmante. Il est jusqu'à un certain point compréhensible que les éducateurs soient portés à percevoir un peu plus positivement le milieu social où vivent les jeunes.

Il est toujours difficile d'objectiver sa situation, de distinguer entre ce qui est et ce qui devrait être. Un point intéressant à noter à ce sujet est la différence de perception entre les jeunes et les éducateurs relativement au contrôle perçu. À Radece, Logatec, Boys' Farm et au Mont Saint-Antoine, les éducateurs perçoivent un contrôle plus faible que les jeunes. La seule exception se trouve à Boscoville, elle est toutefois de peu d'importance puisque l'écart est peu élevé ; ce qui est le cas d'ailleurs à toutes les autres échelles. Le fait que les éducateurs affichent sur la majorité des échelles des moyennes plus élevées que les jeunes et que la seule exception commune à quatre institutions se situe à l'échelle contrôle démontrent sans doute que le contrôle est un aspect considéré négativement en milieu de rééducation.

Il est certain que la perception d'un milieu ne traduit pas toujours parfaitement la réalité concrète de ce milieu. Une perception est naturellement empreinte d'un certain degré de subjectivité. De même, un certain écart de perception entre deux catégories de personnes évaluant le climat social de leur milieu est admissible. Un écart trop accentué est toutefois moins naturel. Aussi, à Boy's Farm les écarts de perception entre les jeunes et les éducateurs, lesquels se traduisent par des variations procentuelles de $33 \%, 26 \%, 43 \%, 27 \%, 25 \%, 32 \%, 14 \%, 19 \%$ et $23 \%$ sur les neuf échelles, sont assez alarmants et laissent supposer l'existence d'assez peu d'entente entre les jeunes et les éducateurs. 


\subsection{Les trois institutions québécoises}

Jusqu'ici nous avons démontré l'utilité d'une mesure du climat social pour évaluer la qualité du travail thérapeute d'une institution et surtout comment l'instrument permettait de différencier les institutions. Dans cette section, nous aborderons la même question mais en termes de différenciation des unités à l'intérieur d'une institution. Les tableaux IV, V et VI décrivent le climat social existant dans chaque unité de vie des trois institutions québécoises, soit 4 unités à Boys' Farm, 5 unités à Boscoville et 10 unités au Mont-Saint-Antoine.

La première remarque qui s'impose est que des différences de climat social n'existent pas uniquement entre institutions. A l'intérieur d'une même institution se trouvent parfois des différences importantes de climat social selon les unités de vie. Cela est particulièrement vérifiable à l'institution Boys' Farm où, en considérant uniquement la perception des jeunes, on note l'existence de deux unités ( 2 et 4 ) où les moyennes enregistrées sont très faibles comparativement aux deux autres. L'unité 4 est celle qui se distingue le plus. Le contrôle y est relativement élevé $(5,81)$, les jeunes se sentent peu engagés $(4,46)$, peu libres de s'exprimer $(5,13)$, peu autonomes $(4,44)$, etc. La situation est d'autant plus inquiétante que les éducateurs de cette unité y évaluent positivement le climat social. Ceci dénote pour le moins une certaine confusion dans l'unité, et nous amène à poser au moins une question : si la perception des jeunes traduit la réalité, comment les éducateurs peuvent-ils y remédier puisqu'ils ne la perçoivent pas ? Les deux autres unités ( 1 et 3 ) présentent des moyennes élevées (de l'ordre de 7,8 et 9 ), ce qui signifie que l'ambiance thérapeutique y est plus marquée.

$\mathrm{Au}$ Mont Saint-Antoine on note également une certaine hétérogénéité entre les unités mais pas de façon aussi marquée qu'à Boys' Farm : les moyennes sont dans l'ensemble entre 6 et 8. Toutefois, dans certaines unités, il existe des écarts considérables de perception entre les jeunes et les éducateurs. Ceci est particulièrement observable aux unités $1,4,8$ et 10 où les écarts varient entre un et trois points. Un fait assez inusité, les éducateurs de l'unité 10 , et surtout de l'unité 3 , affichent sur la majorité des échelles des moyennes de beaucoup inférieures à celles des jeunes. Est-ce parce que les jeunes constituent le principal élément moteur, directeur de l'unité, ce qui amène les éducateurs 
TABLEAU IV

Le climat social dans les 4 unités de vie de Boy's Farm (Moyennes et écarts types)

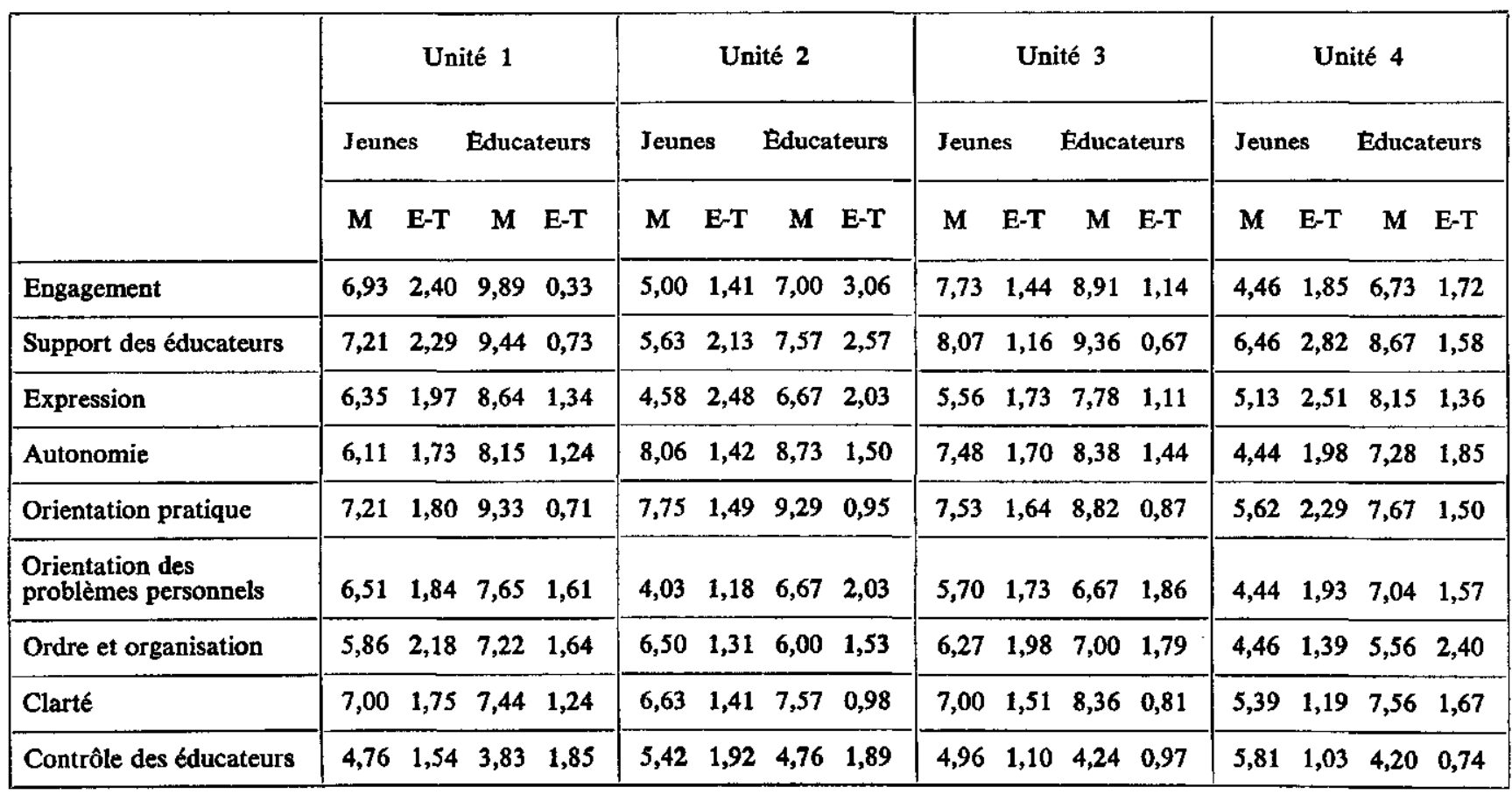


TABLEAU V

Le climat social dans les cinq unités de vie d Boscoville (Moyennes et écarts types)

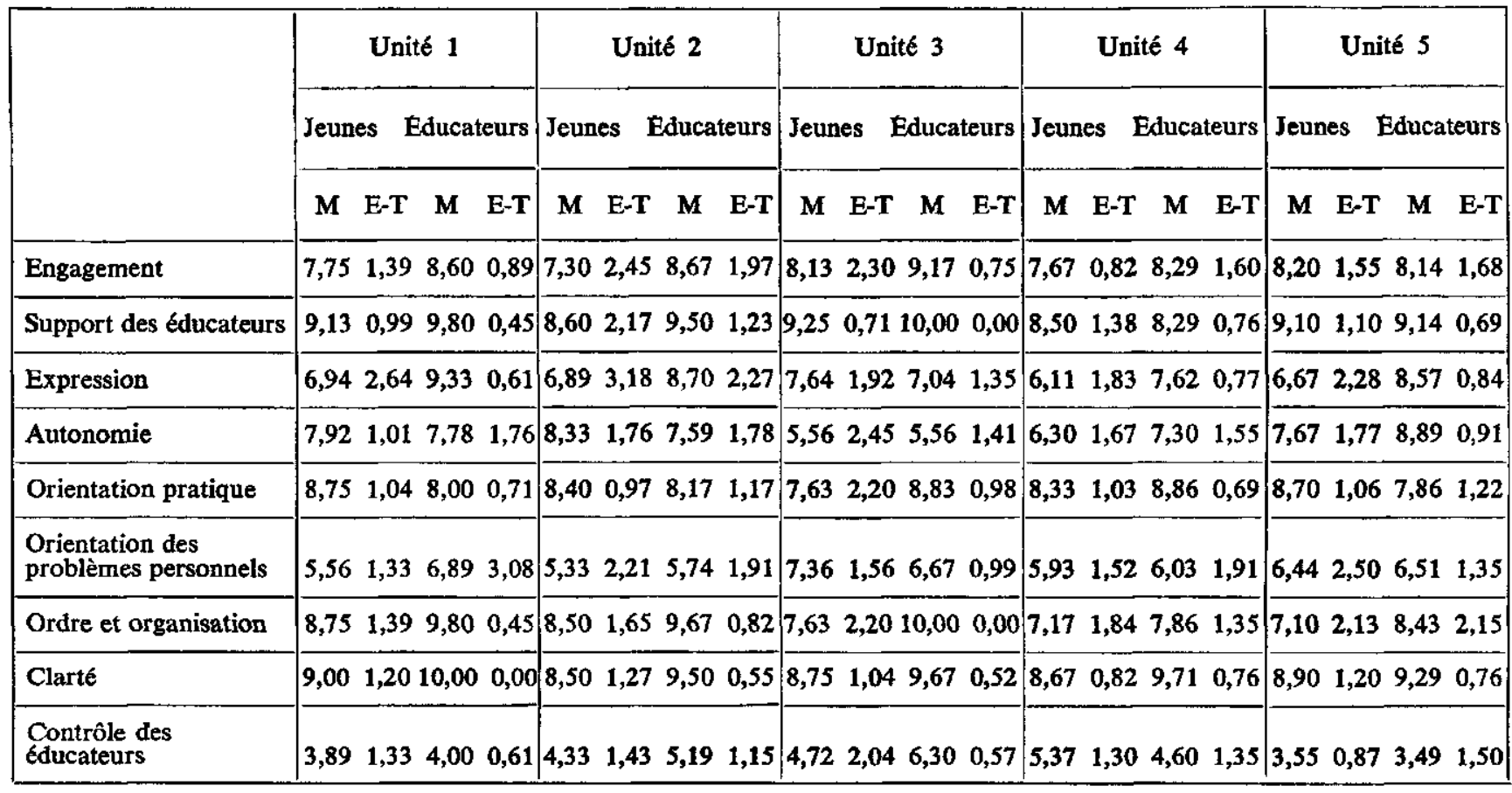


TABLEAU VI

Le climat social dans les 10 unités de vie du Mont-Saint-Antoine (Moyennes)

\begin{tabular}{|c|c|c|c|c|c|c|c|c|c|c|c|c|c|c|c|c|c|c|c|}
\hline & \multicolumn{2}{|c|}{ Unité 1} & Unité 2 & \multicolumn{2}{|c|}{ Unité 3} & \multicolumn{2}{|c|}{ Unité 4} & \multicolumn{2}{|c|}{ Unité 5} & \multicolumn{2}{|c|}{ Unité 6} & \multicolumn{2}{|c|}{ Unité 7} & \multicolumn{2}{|c|}{ Unité 8} & \multicolumn{2}{|c|}{ Unité 9} & \multicolumn{2}{|c|}{ Unité 10} \\
\hline & $\mathbf{J}$ & $\mathbf{E}$ & $\mathbf{E}$ & J & $\mathbf{E}$ & $\mathbf{J}$ & $\mathrm{E}$ & $J$ & $\mathbf{E}$ & & $\mathbf{E}$ & $\mathbf{J}$ & $\mathbf{E}$ & & & & $\mathbf{E}$ & & $\mathbf{E}$ \\
\hline Engagement & 6,71 & 7,00 & $7,82 \quad 8,80$ & 7,11 & 4,20 & 7,70 & 9,60 & 5,07 & 6,50 & 8,07 & 7,40 & 5,40 & 5,83 & 5,08 & 8,14 & 5,52 & 5,14 & 6,34 & 3,67 \\
\hline $\begin{array}{l}\text { Support des } \\
\text { éducateurs }\end{array}$ & 6,57 & 7,80 & $8,598,60$ & 7,78 & 5,60 & 7,55 & 9,40 & 7,54 & 8,33 & 8,36 & 8,00 & 6,80 & 8,00 & 6,33 & 9,14 & 6,52 & 5,57 & 7,17 & 5,50 \\
\hline Expression & 5,56 & 7,56 & $5,62 \quad 7,78$ & 5,68 & 5,56 & 5,39 & 8,00 & 4,28 & 5,56 & 6,67 & 9,56 & 5,11 & 6,67 & 4,03 & 7,14 & 4,87 & 5,24 & 5,05 & 4,63 \\
\hline Autonomie & 6,19 & 8,67 & $6,608,22$ & 6,67 & 5,11 & 6,56 & 8,00 & 5,73 & 7,04 & 6,19 & 7,33 & 5,44 & 6,67 & 5,05 & 7,30 & 5,19 & 6,19 & 5,89 & 4,07 \\
\hline $\begin{array}{l}\text { Orientation } \\
\text { pratique }\end{array}$ & 6,43 & 9,60 & $7,53 \quad 8,40$ & 6,78 & 5,80 & 7,45 & 9,40 & 6,77 & 8,17 & 7,93 & 8,00 & 6,40 & 7,00 & 6,29 & 8,14 & 6,33 & 5,43 & 6,80 & 5,83 \\
\hline $\begin{array}{l}\text { Orientation des } \\
\text { problèmes pers. }\end{array}$ & 5,24 & 5,56 & $5,105,56$ & 6,17 & 5,78 & 4,28 & 6,89 & 5,47 & 5,56 & 5,71 & 6,22 & 5,44 & 5,56 & 5,09 & 6,35 & 5,13 & 5,87 & 5,24 & 3,15 \\
\hline $\begin{array}{l}\text { Ordre et } \\
\text { organisation }\end{array}$ & 6,07 & 9,20 & $7,368,60$ & 5,33 & 5,20 & 7,05 & 9,00 & 6,08 & 8,50 & 6,14 & 6,00 & 6,40 & 5,83 & 5,67 & 7,86 & 6,38 & 5,71 & 6,22 & 9,00 \\
\hline Clarté & 6,00 & 9,80 & $7,659,20$ & 6,00 & 7,20 & 7,00 & 9,00 & 6,39 & 7,50 & 7,50 & 8,60 & 7,20 & 9,00 & 6,96 & 8,57 & 5,48 & 6,29 & 6,64 & 8,50 \\
\hline $\begin{array}{l}\text { Contrôle } \\
\text { éducateurs }\end{array}$ & 5,00 & 2,89 & $4,18 \quad 3,11$ & 6,05 & 5,11 & 4,83 & 2,44 & 4,79 & 4,07 & 5,48 & 4,00 & 6,33 & 2,96 & 6,02 & 4,29 & 5,87 & 3,18 & 5,40 & 5,74 \\
\hline
\end{tabular}


à suivre les événements, et à ne pas apprécier cette situation ? Ainsi donc chaque unité semble avoir sa personnalité propre.

À Boscoville, les jeunes perçoivent le climat social de leur unité respective de façon très similaire. Le climat social à Boscoville est plutôt homogène. De même, il existe dans l'ensemble un consensus général entre les jeunes et les éducateurs. On ne peut pas vraiment ressortir des différences importantes de perception entre jeunes et éducateurs, et ce pour les cinq unités.

Cette section, même si elle n'a pas analysé en profondeur les différences à l'intérieur d'une institution, a quand même démontré que l'instrument pouvait les percevoir et que ces observations pouvaient conduire à des indications précises sur les faiblesses du climat de l'ambiance thérapeutique de chaque unité.

\section{De l'utilité de la mesure du climat social}

Le contrôle opéré par les éducateurs auprès des jeunes semble le déterminant majeur de la nature du régime de vie institutionnelle et une indication de la profondeur du travail rééducatif effectué. Selon les résultats observés dans l'ensemble des institutions américaines, yougoslaves et québécoises à l'étude, à un fort contrôle correspond un faible engagement du jeune à son milieu, un faible support des éducateurs et, il va de soi, une faible liberté d'expression et d'autonomie.

Un fort contrôle peut s'avérer utile dans certaines circonstances. Par exemple, dans une unité d'accueil et d'acclimatation à l'institution un contrôle serré permet d'encadrer le jeune et l'empêche de passer à l'acte sous l'influence de ses impulsions qu'il ne sait encore maîtriser. En somme, un contrôle constant permet de retenir le jeune à l'institution de façon à ce que celui-ci puisse par la suite s'engager dans un processus de rééducation sans trop d'anicroches. Par contre, un tel régime de vie ne peut être appliqué indéfiniment car il ne suscite pas une intégration véritable du jeune à son milieu et à son programme de rééducation, c'est-à-dire une participation active et un engagement personnel dans sa rééducation.

Un contrôle restreint n'est pas nécessairement synonyme de milieu de vie désordonné. Ainsi, à Boscoville le contrôle perçu par les jeunes est très bas, par contre ceux-ci considèrent que leur milieu est bien ordonné et les règles et procédures du 
programme claires. Il ne s'agit donc pas d'un milieu anarchique où les éducateurs ont abandonné leurs responsabilités. Un régime de vie planifié où les éducateurs maintiennent une vigilance et un support constant auprès des jeunes compense, supplée un contrôle rigide qui les étouffe.

Si la première utilité de la mesure du climat social est la caractérisation du milieu, sa seconde utilité est de rendre compte de la communauté d'orientation des jeunes et des éducateurs.

La perception d'un milieu ne correspond pas toujours parfaitement à la réalité concrète de ce milieu. Par exemple, un certain degré de subjectivité peut gauchir la réalité. Toutefois, si l'on accepte l'idée que l'opinion ou la perception qu'un groupe a de son milieu a une importance sinon plus grande, tout au moins égale à la réalité, sur ses attitudes et son comportement, on admet que connaître la perception du groupe de son milieu social est très utile. Dans un milieu de rééducation, la perception des éducateurs du milieu social et celle des jeunes sont également importantes puisque ces deux groupes interagissent continuellement.

La double évaluation du climat social, c'est-à-dire l'évaluation des jeunes et des éducateurs, peut susciter diverses relations entre la perception de ces deux groupes. S'il y a concordance entre leur perception, cette double évaluation permet d'objectiver jusqu'à un certain point des perceptions qui sont empreintes de subjectivité. Elle ajoute à la crédibilité du climat réel mesuré par des perceptions. Il est possible par contre que la perception des jeunes diffère de celle des éducateurs. Par exemple, dans une unité où les jeunes font à toutes fins pratiques ce qu'ils veulent, ceux-ci percevront très positivement leur milieu de vie mais non les éducateurs. L'inverse peut également survenir, à savoir que les jeunes décrivent un climat social très négatif et les éducateurs un climat social très positif. Dans le premier cas, on peut faire l'hypothèse, comme Moos (1973) le suggère, qu'il y a une sousculture institutionnelle présente dans l'unité de l'institution tandis que dans le second cas on peut soutenir que les éducateurs, tout en ayant des objectifs louables, ne sont pas conscients de la réalité perçue par les jeunes; ils définissent un stéréotype de rééducation.

Néanmoins, le simple fait de constater une divergence fait prendre conscience qu'un malaise existe. Celui-ci peut être cor- 
roboré par d'autres observations ou tout au moins servir de point de départ à une investigation approfondie. Cette information pourra permettre une intervention avant que la situation ne se soit tellement détériorée qu'elle saute aux yeux et que la solution ne devienne problématique.

Cette notion d'auto-évaluation, d'auto-contrôle, conduit à une troisième utilité de l'instrument de mesure du climat social. En effet, si on l'utilise de façon continue, il permet de tracer l'évolution d'un milieu et ainsi de porter un jugement sur l'adéquacité des tendances observées compte tenu des objectifs de l'unité et de l'institution.

Le climat social perçu par les jeunes et les éducateurs constitue donc un indicateur précieux, tant au point de vue clinique qu'administratif. Il est une source d'informations appréciables sur le type de régime de vie et sur la nature du programme de rééducation. La connaissance du climat social permet de déceler les points forts et/ou faibles d'une unité de rééducation et, éventuellement, d'intervenir si la situation l'exige. C'est un outil d'évaluation dont l'utilisation est facile.

\section{BIBLIOGRAPHIE}

BRILL, R., DUNCAN, B. (1977), Staff team climates and treatment unit environment, G.R.I.J. Technical report $\mathrm{n}^{\mathrm{o}} 4$, Montreal.

CAPUL, M. (1969), les Groupes réducatifs, Paris, Presses Universitaires de France.

EMPEY, L.T., LUBECK, S.G. (1971), The Silverlake experiment, Chicago, Aldine.

GERST, M., MOOS, R. (1972), «The social ecology of university student residences \$, Journal of Educational Psychology, 63, 513-525.

JESNESS, C.F. (1962), The Fricot Ranch school study: an analysis of a training school experience, Fricot progress report No. 3, California Youth Authority, Division of Research.

MAGNUSSON, D., GERZEN, M., NYMAN, B. (1968), * The generality of behavioral date I : generalization from observations on one occasion \#, Multivariate Behavioral Research, 3, 295-320.

MENARD, R. (1976), le Climat social dans une institution pour jeunes délinquants : Boscoville, Montréal, G.R.I.J., rapport technique, no 13.

MISCHEL, W. (1968), Personality and assessment, New York. Wiley.

MOOS, R.H. (1969), \& Sources of variance in responses to questionnaires and in behavior *. Journal of Abnormal Psychology, 74, 405-412.

MOOS, R.H. (1973), Correctional Institutions Environment Scale, California, Social Ecology Laboratory. 
MOOS, R., HUMPHREY, B. (1973), Group Environment Scale, California, Social Ecology Laboratory.

SELOSSE, J. et al. (1972), l'Internat de rééducation, Paris, Cujas.

VODOPIVEC, (1974), Maladjusted Youth: an experiment in rehabilitation, Farnborough, Saxon House.

WENK, E., MOOS, R.H. (1972), \& Social climates in prisons $\gg$, Journal of Research in Crime and Delinquency, 9, 134-148. 